\title{
On the Exact Solution of the Mixed-Spin Ising Chain with Axial and Rhombic Zero-Field Splitting Parameters
}

\author{
M. DANČO AND J. STREČKA
}

Department of Theoretical Physics and Astrophysics, Faculty of Science, P.J. Šafárik University

Park Angelinum 9, 04001 Košice, Slovak Republic

Ground-state phase diagram of the mixed spin-1/2 and spin-1 Ising chain with axial and rhombic zero-field splitting parameters is exactly calculated within the framework of the transfer-matrix method. It is shown that the rhombic zero-field splitting parameter prefers the magnetically ordered phase instead of the disordered phase.

PACS numbers: 05.50.+q, 05.70.Jk, 75.10.Pq

\section{Introduction}

Exactly solved one-dimensional quantum spin models traditionally belong to the most fascinating research areas as they provide valuable insight into otherwise hardly understandable aspects of cooperative and quantum phenomena [1]. In this work, we will exactly treat the mixed spin-1/2 and spin-1 Ising chain with axial and rhombic zero-field splitting parameters.

\section{Model and its exact solution}

Consider the Ising model for a chain consisting of the alternating spin- $1 / 2$ and spin- 1 atoms, which accounts also for axial and rhombic zero-field splitting (ZFS) parameters. The total Hamiltonian of this spin system can be written as a sum of two terms $\hat{\mathcal{H}}=\hat{\mathcal{H}}_{\text {ex }}+\hat{\mathcal{H}}_{\text {zfs }}^{(1)}$. The former term accounts for the Ising-type exchange interaction between the nearest-neighbor spins

$$
\hat{\mathcal{H}}_{\mathrm{ex}}=-J \sum_{k=1}^{N} \hat{S}_{k}^{z}\left(\hat{\sigma}_{k}^{z}+\hat{\sigma}_{k+1}^{z}\right),
$$

and the latter term accounts for the axial $(D)$ and rhombic $(E)$ ZFS parameters acting on the spin-1 atoms only

$$
\hat{\mathcal{H}}_{\mathrm{zfs}}^{(1)}=-D \sum_{k=1}^{N}\left(\hat{S}_{k}^{z}\right)^{2}-E \sum_{k=1}^{N}\left[\left(\hat{S}_{k}^{x}\right)^{2}-\left(\hat{S}_{k}^{y}\right)^{2}\right] .
$$

Above, $\hat{\sigma}_{k}^{z}$ and $\hat{S}_{k}^{\alpha}(\alpha=x, y, z)$ denote standard spatial components of the spin- $1 / 2$ and spin- 1 operators, respectively, $N$ denotes a total number of spin- $1 / 2$ (spin-1) atoms and the periodic boundary condition $\sigma_{\mathrm{N}+1} \equiv \sigma_{1}$ is imposed for further convenience. It is worthwhile to remark that there is one-to-one correspondence between the Hamiltonian $\hat{\mathcal{H}}_{\mathrm{zfs}}^{(1)}$ given by Eq. (2) and the Hamiltonian with three different parameters $D^{x}, D^{y}$ and $D^{z}$

$$
\hat{\mathcal{H}}_{\mathrm{zfs}}^{(2)}=-D^{x} \sum_{k=1}^{N}\left(\hat{S}_{k}^{x}\right)^{2}-D^{y} \sum_{k=1}^{N}\left(\hat{S}_{k}^{y}\right)^{2}-D^{z} \sum_{k=1}^{N}\left(\hat{S}_{k}^{z}\right)^{2} .
$$

The equivalence between $\hat{\mathcal{H}}_{\mathrm{zfs}}^{(1)}$ and $\hat{\mathcal{H}}_{\mathrm{zfs}}^{(2)}$ can easily be verified by establishing a rigorous mapping correspondence between the relevant interaction terms appearing in the Hamiltonians (2) and (3). The total angular momentum of the spin-1 atoms is integral of motion $\hat{\boldsymbol{S}}_{k}^{2}=\left(\hat{S}_{k}^{x}\right)^{2}+\left(\hat{S}_{k}^{y}\right)^{2}+\left(\hat{S}_{k}^{z}\right)^{2}=2$ and hence, one of three parameters $D^{x}, D^{y}$ and $D^{z}$ must depend on the other two. Consequently, the Hamiltonians $\hat{\mathcal{H}}_{\text {zfs }}^{(1)}$ and $\hat{\mathcal{H}}_{\mathrm{zfs}}^{(2)}$ differ one from the other just by some constant factor $\hat{\mathcal{H}}_{\mathrm{zfs}}^{(1)}=\hat{\mathcal{H}}_{\mathrm{zfs}}^{(2)}+C$, whereas the relevant interaction terms $C, D$ and $E$ are connected to the ones $D^{x}, D^{y}$ and $D^{z}$ through the mapping relations

$$
\begin{aligned}
& C=D^{x}+D^{y}, \quad D=D^{z}-\frac{D^{x}+D^{y}}{2}, \\
& E=\frac{D^{x}-D^{y}}{2} .
\end{aligned}
$$

The model under investigation thus turns out to be equivalent to the one recently studied by $\mathrm{Wu}$ et al. [2] using the approach based on the Jordan-Wigner transformation.

Here, the investigated model system will be exactly treated within the framework of transfer-matrix method [3]. First, it is useful to rewrite the total Hamiltonian as a sum of site Hamiltonians $\hat{\mathcal{H}}=\sum_{k} \hat{\mathcal{H}}_{k}$, whereas each site Hamiltonian $\hat{\mathcal{H}}_{k}$ involves all the interaction terms associated with the spin- 1 atom from the $k$-th lattice site

$$
\begin{aligned}
& \hat{\mathcal{H}}_{k}=-J \hat{S}_{k}^{z}\left(\hat{\sigma}_{k}^{z}+\hat{\sigma}_{k+1}^{z}\right)-D\left(\hat{S}_{k}^{z}\right)^{2} \\
& -E\left[\left(\hat{S}_{k}^{x}\right)^{2}-\left(\hat{S}_{k}^{y}\right)^{2}\right] .
\end{aligned}
$$

Due to a validity of commutation relation between different site Hamiltonians, the partition function can be partially factorized into the product

$$
\mathcal{Z}=\sum_{\left\{\sigma_{k}\right\}} \prod_{k=1}^{N} \operatorname{Tr}_{S_{k}} \exp \left(-\beta \hat{\mathcal{H}}_{k}\right)
$$

where $\beta=1 / k_{\mathrm{B}} T, k_{\mathrm{B}}$ is Boltzmann's constant, $T$ is the absolute temperature, $\operatorname{Tr}_{S_{k}}$ means a trace over spin degrees of freedom of the $k$-th spin-1 atom and $\sum_{\left\{\sigma_{k}\right\}}$ de- 
notes a summation over all possible configurations of the spin-1/2 atoms. After tracing out spin degrees of freedom of the spin-1 atom, the relevant expression on right hand side of Eq. (6) will depend just on its two nearest-neighbor spins $\sigma_{k}$ and $\sigma_{k+1}$. Moreover, this expression can be subsequently used in order to define the transfer matrix

$$
\begin{aligned}
& T\left(\sigma_{k}, \sigma_{k+1}\right)=\operatorname{Tr}_{S_{k}} \exp \left(-\beta \hat{\mathcal{H}}_{k}\right)=1 \\
& \quad+2 \exp (\beta D) \cosh \left(\beta \sqrt{J^{2}\left(\sigma_{k}^{z}+\sigma_{k+1}^{z}\right)^{2}+E^{2}}\right) .
\end{aligned}
$$

The rest of our exact calculations can be accomplished using the standard procedure developed within the transfer-matrix approach [3]. This rigorous technique allows one to express the partition function in terms of respective eigenvalues of the transfer matrix

$$
\mathcal{Z}=\sum_{\left\{\sigma_{k}\right\}} \prod_{k=1}^{N} T\left(\sigma_{k}^{z}, \sigma_{k+1}^{z}\right)=\operatorname{Tr} T^{N}=\lambda_{+}^{N}+\lambda_{-}^{N} .
$$

In the thermodynamic limit $N \rightarrow \infty$, the free energy per unit cell can be expressed solely in terms of the largest eigenvalue of the transfer matrix

$$
f=-k_{\mathrm{B}} T \lim _{N \rightarrow \infty} \frac{1}{N} \ln \mathcal{Z}=-k_{\mathrm{B}} T \ln \left(T_{11}+T_{12}\right),
$$

where $T_{11}=T( \pm 1 / 2, \pm 1 / 2)$ and $T_{12}=T( \pm 1 / 2, \mp 1 / 2)$ were used to denote two different matrix elements of the transfer matrix defined through Eq. (7).

\section{Results and discussion}

Now, let us take a closer look at the ground-state behavior of the investigated model system. For simplicity, our subsequent analysis will be restricted only to the particular case with the ferromagnetic interaction $J>0$, since the relevant change in sign of the parameter $J$ causes just a rather trivial reversal of all the spin- $1 / 2$ atoms.

In the zero temperature limit, the first-order phase transition line given by the condition $D=-\sqrt{J^{2}+E^{2}}$ separates the ferromagnetically ordered phase (OP) from the disordered phase (DP). The relevant spin order appearing in the OP and DP can be unambiguously defined through the eigenvectors

$$
\begin{aligned}
& |\mathrm{OP}\rangle=\otimes_{k}|1 / 2\rangle_{k}\left[\cos \left(\frac{\varphi}{2}\right)|+1\rangle_{k}+\sin \left(\frac{\varphi}{2}\right)|-1\rangle_{k}\right], \\
& |\mathrm{DP}\rangle=\otimes_{k}| \pm 1 / 2\rangle_{k}|0\rangle_{k},
\end{aligned}
$$

where the product runs over all lattice sites, the former (latter) ket vectors specify the state of the spin- $1 / 2$ (spin-1) atoms and the mixing angle $\varphi$ is given by $\varphi=$ $\arctan (E / J)$. In the DP, all the spin-1 atoms tend toward their "non-magnetic" spin state $|0\rangle$ on behalf of a sufficiently strong (negative) axial ZFS parameter and hence, each spin- $1 / 2$ atom may completely independently choose any of two available spin states $| \pm 1 / 2\rangle$. However, the more striking spin order emerges in the OP, where the magnetic behavior of the spin- 1 atoms is governed by a quantum entanglement of two magnetic spin states $|+1\rangle$ and $|-1\rangle$ and all the spin- $1 / 2$ atoms reside their "up" spin state $|1 / 2\rangle$. In this respect, the rhombic ZFS parameter gradually destroys a perfect ferromagnetic order between the spin- $1 / 2$ and spin- 1 atoms, which appears in an absence of the rhombic term.
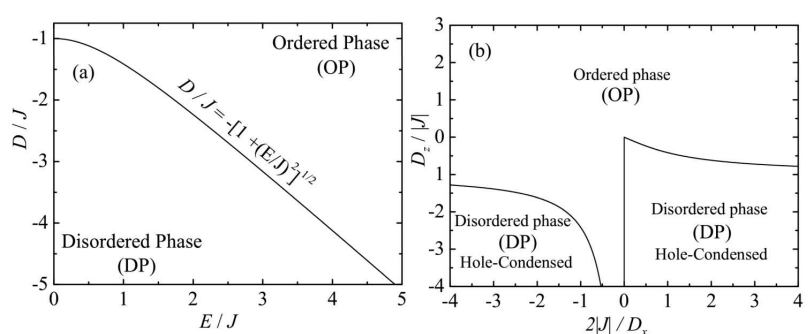

Fig. 1. Ground-state phase diagram in two different planes: (a) $E / J-D / J$ plane; (b) $2 J / D_{x}-D_{z} / J$ plane for $D_{y}=0$.

For better illustration, Fig. 1a depicts the ground-state phase diagram in the $E / J-D / J$ plane. The most surprising finding stemming from Fig. 1a is that the phase boundary between $\mathrm{OP}$ and DP shifts toward more negative values of the axial ZFS parameter when increasing a strength of the rhombic ZFS parameter. Accordingly, it turns out that the quantum entanglement between the spin states $|+1\rangle$ and $|-1\rangle$, which is caused solely by the rhombic ZFS parameter, energetically stabilizes the OP before the DP. For comparison, Fig. 1b illustrates the ground-state phase diagram in the $2 J / D_{x}-D_{z} / J$ plane when using Eq. (3) in order to define the ZFS Hamiltonian. Let us note that this phase diagram is in accord with the recent results of $\mathrm{Wu}$ et al. [2], but this phase diagram is apparently less convenient for interpreting the phase boundary between OP and DP as the parameter $D_{x}$ changes according Eq. (4) both axial as well as rhombic ZFS parameters.

In conclusion, it is worthy to notice that the rigorous procedure developed on the grounds of the transfer-matrix method can readily be adapted to treat the investigated model system even in a presence of non-zero external magnetic field, which will be examined in detail in our forthcoming work.

\section{Acknowledgments}

This work was supported under the grant VEGA $1 / 0128 / 08$

\section{References}

[1] D.C. Mattis, The Many-Body Problem: An Encyclopedia of Exactly Solved Models in One Dimension, World Scientific, Singapore 1993.

[2] H. Wu, G. Wei, G. Yi, Phys. Lett. A 372, 6531 (2008).

[3] H.A. Kramers, G.H. Wannier, Phys. Rev. 60, 252 (1941). 